PRE-PRINT: Briscoe, A., M. A. Strickland, et al. (2008). Medial-lateral Loading and Wear in Total Knee Arthroplasty. 16th Congress, European Society of Biomechanics. Lucerne, Switzerland. Abstracts published in J. Biomechancs 41, Supplement 1 (Jul 2008) pS236 DOI: 10.1016/S0021-9290(08)70235-5

\title{
MEDIAL-LATERAL LOADING AND WEAR IN TKA
}

\author{
Adam Briscoe ${ }^{1}$, Michael Strickland ${ }^{1}$, Mark Taylor ${ }^{1}$ \\ 1Bioengineering Sciences Research Group, University of Southampton, UK
}

\section{Introduction}

Pre-clinical wear testing of Total Knee Arthroplasty has traditionally been carried out in a physical experiment [Fisher, 2002]. Recent computational models have been shown to have sufficient accuracy to be considered alongside these experiments [Knight, 2007]. These computational models use a relationship of wear volume proportional to the product of contact pressure, sliding distance and cross shear [Maxian, 1996]. Instrumented knees have recently shown that medial-lateral (ML) loads may be of similar magnitudes to that of the anterior-posterior (AP) load. The AP load is known to have a significant effect on the kinematics of the total knee replacement and so it is reasonable to assume that application of an ML load may have a similar degree of influence on kinematics. The effect of the ML load is hypothesised to increase the cross shear and hence the wear rate. At present, the ISO standard for testing TKA contains no provision for a ML load [BS ISO, 2002].

\section{Method}

Geometry for the PFC $\Sigma$ CVDC, PFC $\Sigma$ PLIC and NexGen $\odot$ total knee replacements were generated and placed into a virtual Stanmore Knee Simulator, configured as in the literature [Knight, 2007]. For a second set of models, M-L load values were added having been adapted from the literature for a 70kg patient [Godest, 2002] and applied to the tibial component. All loads and the flexion angles applied are shown in Figure 1. Each model was run for a level gait cycle and then local wear depth was calculated. Each element was then displaced inferiorly, assuming linear wear over one million cycles. The process was then repeated up to 8 million cycles.

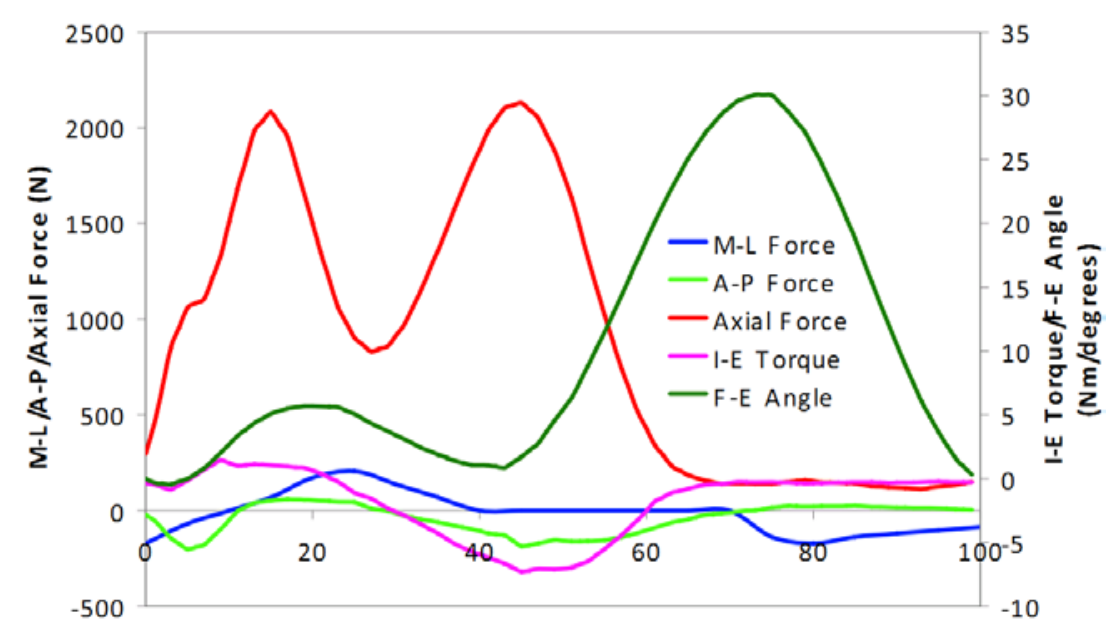

$\%$ G ait Cycle

Figure 1: Input kinetics for normal gait. 'Positive' is anterior, lateral and internal. 
2 Each of the three designs showed an increase in the volumetric wear rate when an ML load was included. The 3 percentage increase varied from $72 \%$ for the PLIC geometry to $177 \%$ for NexGen@. Inclusion of the ML load 4 resulted in a broader wear scar and greater wear depths for all three knee designs (Figure 2).

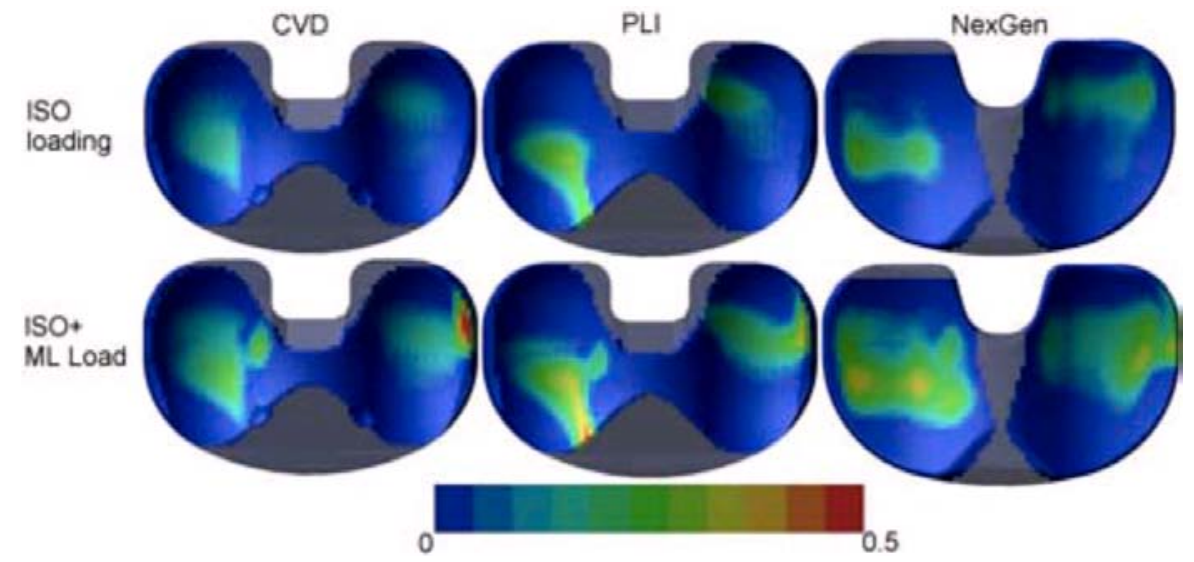

7 Figure 2: Contour Plots of wear scars produced after 8 million cycles. Contours depict the linear wear depth in $\mathrm{mm}$.

\section{Discussion}

10 The effect of including an ML load was to increase the volumetric wear rate. In all cases the amount of volumetric

11 wear produced, increased significantly. This increase in wear was attributed to an increase in cross-shear between

12 the two surfaces. Cross shear is calculated as the ratio between AP and ML sliding distance. By applying an ML force

13 the component of sliding in the ML direction was increased and so cross shear increased. The percentage increase

14 was not constant for all designs and so ranking under ISO loads and ISO+ML loads may not be identical. It is

15 therefore shown that ML loads may be important when modelling wear in TKA.

\section{Acknowledgements}

18 Research supported by DePuy, a Johnson \& Johnson company.

\section{References}

BS ISO 14243-1, 2002.

Fisher J. et al, Proc Inst Mech Eng (H), 215(2): 127-132, 2001. 